\title{
Mesoscopic simulations of plastic deformation
}

\author{
B. Devincre*, L.P. Kubin, C. Lemarchand, R. Madec \\ LEM, CNRS-ONERA, 29 Av. de la Division Leclerc, BP 72, 92322 Châtillon Cedex, France
}

\begin{abstract}
This review is focused on recent progress achieved by mesoscopic simulations of plastic deformation. The methods presently available for discretizing the dislocation lines are critically discussed with emphasis on a new lattice-based model. Progress in large-scale simulations is represented by a study on the influence of long range elastic stresses on the formation of dislocation patterns in fcc crystals. A hybrid discrete-continuum method that provides an exact treatment of the boundary conditions is described and illustrated by an investigation of the critical conditions for dislocation motion in the channels of $\gamma / \gamma^{\prime}$ superalloys. (c) 2001 Elsevier Science B.V. All rights reserved.
\end{abstract}

Keywords: Simulations; Dislocation patterns; Dislocation dynamics; Cross-slip

\section{Introduction}

The idea of simulating the dynamics and interactions of dislocation lines in an elastic continuum in order to study the plastic crystals is now 40 years old. In the early times, simulation methods have been applied to rather simple situations, for instance the motion of a single dislocation line through a random distribution of point obstacles representing either forest dislocations or a distribution of small precipitates ([1-3]). In the last 10 years, a substantial improvement of the range and realism of such simulations has been made possible by the increase in computing power. Thus, today, one can compute the collective properties of large numbers of dislocations (e.g. several hundreds of lines) in sufficiently large model crystals, with typical linear dimension of about $15 \mu \mathrm{m}$. This allows performing a direct comparison between real and in silico experiments. The most important achievement in this domain is certainly the development of three-dimensional simulations accounting for crystallographic effects and slip geometry. Initiated by Kubin and Canova [4-6], these simulations are now reasonably mature. The simulation codes that exist to date can be classified into two different types, depending on whether dislocation dynamics and reactions are simulated on a discrete lattice or in the continuum. Each approach has its respective strengths and weaknesses. In short, the use of an underlying lattice significantly improves the computing efficiency and is therefore, better suited for the modeling of large strains and/or large volumes. In contrast, continuous models describe more

\footnotetext{
${ }^{*}$ Corresponding author. Tel.: +33-1-46734449; fax: +33-1-467334155. E-mail address: devincre@zig.onera.fr (B. Devincre).
}

accurately the elastic fields near the dislocation cores and are usually preferred for a detailed analysis of elementary configurations.

Interesting results have been obtained in both cases. As far as discrete simulations are concerned, this includes the mechanisms of strain hardening in fcc and bcc metals [7-9], the formation of plastic zones close to a crack tip or under an indenter [10,11], the yield point properties of silicon crystals [12] and the flow stress anomaly in the intermetallic alloy $\mathrm{Ni}_{3} \mathrm{Al}$ [13]. Important studies performed with the help of continuous simulations involve the plastic relaxation of internal stresses in thin layers [14], the study of sessile dislocation junctions $[15,16]$ and the interaction of dislocations with small glissile defect clusters $[17,18]$.

The present paper does not aim at reviewing all these achievements. Rather, the objective is to draw an instantaneous picture of a few persisting problems and of some solutions under development. The contents are organized as follows. Section 2 is devoted to the question of simulation efficiency and to a short presentation of a new type of discrete simulation. Section 3 reports on the contribution of long range dislocation interactions to strain hardening and dislocation patterning in fcc metals. These results are important from a theoretical viewpoint but also from a technical one, since the calculation of these interactions constitutes the most intensive part of 3D simulations in terms of CPU time. Section 4 discusses the exact treatment of the boundary conditions and the presently existing solutions. As an illustration, the simulation of dislocation glide in the $\gamma$ channels of single crystal superalloys is reported in Section 5. Finally, concluding remarks are presented in Section 6. 


\section{Simulations of dislocation dynamics}

As several computer models have been published $[6,19,20-23]$, it is possible to discuss the similarities and differences between them. In a first step, all of them consider dislocations as elastic defects embedded into an elastic continuum and, therefore, are principally based on the elastic theory of dislocations. Hence, the force on each dislocation is obtained as minus the gradient of the total elastic energy for the configuration considered. The total elastic energy usually has two different origins: the dislocation fields and the fields at the internal and external boundaries. The dissipation of energy by the moving dislocations, for instance by their interaction with electrons and phonons, is accounted for with the help of a linear relationship:

$v=\frac{\tau^{*} b}{B}$

where $\tau^{*}$ is the resolved effective stress, $b$ the Burgers vector and $B$ a constant viscous drag coefficient. It must be noted that while there is general agreement on Eq. (1) in the case of materials with high dislocation mobility, various relations have been used for materials with high lattice friction or Peierls stress. In such cases, an Arrhenius form is certainly the most adequate one to account for the stress-assisted and thermally activated process of kink pair nucleation $[10,24,25]$.

The most important difference between the various computer models certainly resides in the topological solution used to discretize the dislocation lines. One may make use of an underlying simulation lattice to tile the elastic continuum. Then, the characters of the dislocation segments are restricted to a finite set $[6,19,20]$. Alternatively, the dislocation lines are decomposed into a finite set of straight or curved segments whose orientations are not discretized, connected by nodes at which the forces are computed [21-23]. Identical mathematical expressions for the self-stress fields of the segments can be used in both types of simulations.

At first sight, the discretization procedure used in a "nodal" or quasi-continuous model may seem to be the most attractive one since it provides an accurate description of a curved dislocation line with a minimum number of segments. Indeed, in simulations based on a lattice, the line curvature can only be approximated since the number of segment directions is finite (cf. Fig. 1). The difference is usually of minor importance as far as dislocation dynamics is concerned, but it may become significant when an accurate estimate of the stress is required close to a dislocation line [26]. Nevertheless, this advantage of quasi-continuous models is not decisive for several reasons. First, in several classes of materials, for instance bcc or covalent dc crystals, there is a high lattice friction and the dislocation lines are confined to a few preferential crystallographic directions. Then, a simulation based on a lattice provides an easy way to account for such dislocation core properties. In addition, it allows writing consistent mobility laws, as in such



Fig. 1. A Frank-Read source within the pure-mixed discrete model that incorporates line directions of screw, edge and $\pm 60^{\circ}$ characters.

materials the probability for kink pair nucleation is proportional to the total length of the segment considered. Finally, the use of lattice-based simulation generally results in a substantial improvement of the computer code efficiency for several reasons. It simplifies the problem of the segments interconnectivity, as the connecting operations can be tabulated. It introduces a length scale, the elementary lattice parameter that fixes a border between the elastic and core properties. This is helpful from a numerical point of view in order to identify the situations where core reactions take place. Lastly, many other operations can be tabulated.

Additional differences between the various dislocation dynamics (DD) simulation codes obviously stem from the rules used to model the dislocation core properties. This is a key point, as simulations not accounting for these properties should not be regarded as simulations of plasticity but rather as simulations of dislocation dynamics during a small free-flight time interval. At low and intermediate temperatures, two properties are critical. The first one is dislocation cross-slip, a mechanism that allows the screw dislocations to relax internal stresses thus, promoting dynamic recovery. All the simulations accounting for this mechanism use a set of local rules proposed by Kubin et al. [6,19], which derive from the Friedel-Escaig model.

The second important core property that must be explicitly included is the intersection of dislocations. This mechanism is a priori a complex one, since it involves both long range elastic interactions and interactions at the level of the dislocation cores. However, it has been known for a long time and confirmed recently that the core contribution to those reactions is negligible in energetic terms, so that intersections can be treated in first approximation as a purely elastic problem [16,27]. A consensual idea for dealing with this question is, again, to make use of a minimum set of rules in order to mimic the reactions between dislocations. Three complementary types of rules exist. First, one has to 


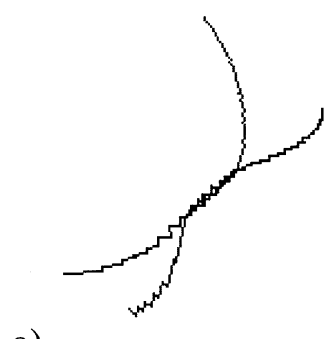

a)

b)

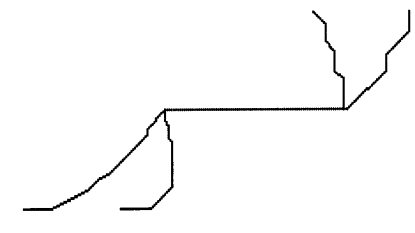

Fig. 2. A junction between two dislocations. (a) Within the edge-screw model. (b) Within the pure-mixed model. Notice the large difference in the number of elementary segments needed to treat the same configuration in the two models.

anticipate the formation of a sessile junction that will anchor the dislocation lines. There, it not easy to set up a simple rule since the reactions strongly depend on the configuration of the lines close to the intersection region but also far from it. It has been shown recently that a simplified rule based on the Frank's criterion leads to a very poor description of the reality $[15,16]$. Precise expressions accounting for the characters of the intersecting dislocations are now preferred, in the case of lattice-based simulations, this leads to reaction predictions that are tabulated once and/or ever. The second set of rules deals with the zipping and unzipping of the sessile junctions. In order to correctly reproduce these mechanisms, one has to account for the elastic interaction forces that tend to align and move the two intersecting dislocations in the direction of the junction, i.e. along the intersection of their respective glide planes. This direction not being usually parallel to the screw or edge direction, the modeling of the junction geometry and strength has been from the beginning a problem for the lattice-based simulations. Indeed, those simulations containing only the edge and screw directions, junctions can then only be described by a knitting of short segments (Fig. 2a), conditionally blocked, with a local destruction stress that depends on the configuration geometry [7]. This problem is now solved and the treatment of the zipping and unzipping of the junctions is much the same in all types of simulations. This important progress of the lattice-based simulations is the outcome of a new topological line model, which will be further, discussed below. In this model, additional line directions, that include in particular the directions of all the possible junction products, are introduced into the initial vector basis used within the simple edge-screw model (Fig. 2b). Finally, a third set of rules may be used to model the defects nucleated along the dislocation lines after their intersection, viz. jogs and kinks of atomic height. It must be emphasized that the modeling of such atomic defects at mesoscale is an unsolved problem. Their dimension is, indeed, far too small compared to the scale of DD simulations in which the elementary length of the dislocation segments must be larger than a few Burgers vectors. Besides, their possible influence on the flow stress and strain hardening properties, mainly in fcc crystals, is

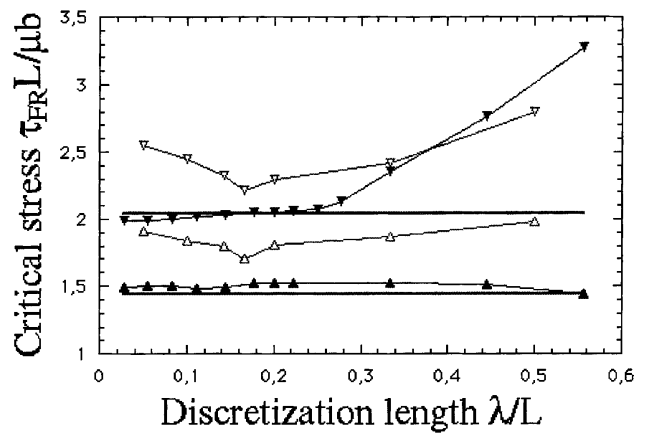

Fig. 3. Critical Frank-Read stress for a source segment of length $L$, in reduced units vs. the reduced discretization length $(\mu$ is the shear modulus). The upper and lower horizontal lines represent the classical elastic solution for screw and edge source segments, respectively. The open triangles show the results obtained with the edge-screw model. An optimum is obtained when the discretization length is about one fifth of the initial segment length. The full triangles show the results obtained within the pure-mixed model. A much better accuracy is obtained up to larger discretization lengths, i.e. with a smaller number of segments.

still the object of debates. This is why quite diverging solutions are adopted in the literature. Either the occurrence of atomic kinks or jogs is simply neglected [8], or the latter are considered as strong pinning points whose strength is determined from the formation energy of vacancies and interstitials [28]. Atomistic simulations would certainly be of great help in assessing the validity of these two opposite views.

To conclude this critical review on the progress of the DD simulation technique by a specific example, the new topological line model for lattice-based simulations is further discussed. The approach achieved appears as a good compromise between the accuracy of the quasi-continuous simulations and the numerical efficiency of the lattice-based simulations. Indeed, by increasing the number of segment directions needed to model a fcc crystal from 24 (within the simplest edge-screw model) to 48 (including two additional mixed orientations), one can significantly reduce the number of pair interactions to be computed. This is simply due to the fact that with additional line directions, dislocation reactions (Fig. 2b), dipolar configurations of mixed character and the curvature of single lines can be modeled with a smaller number of segments. Fig. 3 illustrates the significant improvement in numerical accuracy obtained with the pure-mixed model in the case of the critical stress for the operation of a Frank-Read source.

\section{Long range interactions and dislocation patterning}

Examples of numerical simulations on fcc crystals are given in this section, as an illustration of the progress achieved in the past few years by large-scale DD simulations. The problem treated is concerned with the identification of the properties that govern dislocation patterning during monotonic deformation and their relation to strain 
hardening. These calculations are also of great interest from a technical point of view since they focus on the contribution of the long range elastic interactions of dislocations, which is the most time consuming part of DD simulations.

It has been known since the first transmission electron microscopy observations that dislocation cell structures occur, not only during stage III of the deformation of single crystals, but also from the onset of plastic flow in single crystals deformed in multislip conditions or in the grains of deformed polycrystals. Simulations of areal glide performed in the presence of cross-slip, has shown that two phenomena occur when a gliding dislocation meets a high local stress field [7]. The dislocation line can undergo double cross-slip, which results in dislocation multiplication and increases the density of forest obstacles for non-coplanar slip systems. In addition, some parts of the line can be transformed into three-dimensional immobile debris through the cross-slip of their screw portions. This debris play the role of anchoring points from which an organized microstructure further emerges. From this, it can be concluded that cross-slip is the main mechanism inducing slip irreversibility and dislocation storage.

This scenario has been confirmed by full 3D numerical experiments conducted on [1 000$]$ model fcc crystals. It was checked that, in the absence of cross-slip, no dislocation storage occurs during plastic deformation. As a consequence, strain hardening vanishes and the dislocation density appears to be homogeneously distributed in the crystal. When cross-slip is switched on in the simulation, storage occurs due to the production of small, stable, 3D debris, and an increase of the multiplication rate due to double cross-slip events is observed. As a consequence, strain hardening achieves normal values and a preliminary stage of the formation of dislocation cells can be observed [29,30]. It must be mentioned that cross-slip being a stress-assisted mechanism, it only relaxes the local peaks of internal stress. In other words, it is a mechanism of dynamic recovery. Thus, the classical idea according to which patterning is associated with dynamic recovery by cross-slip [31] does make sense even at low temperatures.

Complementary simulations have been performed in order to estimate the respective contributions of the longand short-distance elastic interactions of dislocations to flow stress and dislocation patterning. To differentiate these two contributions, a virtual cylinder of interaction, of radius $1 \mu \mathrm{m}$ has been introduced around each dislocation segment. Interactions with segments inside or outside this cylinder were considered respectively as short-distance or long-distance ones. Then, long and short-distance elastic interactions were alternatively excluded from the computation of the dislocation interaction forces and dynamics. In both cases, rules governing the formation and destruction of junctions and the local line tension effects were however, not affected.

Two uniaxial tensile tests were simulated on the same [ $\left.1 \begin{array}{ll}1 & 0\end{array}\right]$ model fcc crystal of linear dimension $15 \mu \mathrm{m}$ with

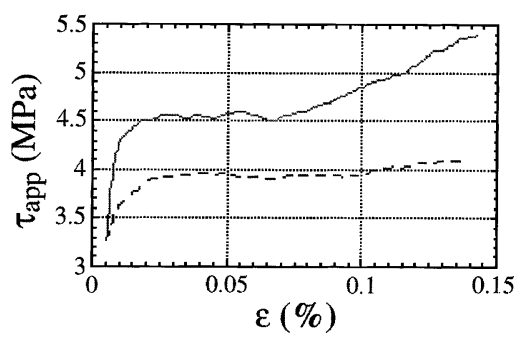

Fig. 4. Simulated stress vs. plastic strain curves for a model [1 000$] \mathrm{fcc}$ crystal. The upper curve is obtained by excluding all the elastic interactions of the dislocation segments outside a cut-off cylinder of $1 \mu \mathrm{m}$. The lower (dotted) curve is the complementary of the previous one, where all the short-distance interactions inside the cylinder are omitted.

only the long- or short-distance interactions being accounted for.

The corresponding stress versus strain curves are shown in Fig. 4. With respect to a full calculation taking into account all the elastic interactions between the dislocation segments the yield stresses do not appear to be strongly affected, particularly when long range interactions are neglected. This simply confirms that junction unzipping combined with line tension effects provide the major contribution to the yield stress of fcc crystals. A first noticeable difference between the two stress-strain curves of Fig. 4 is a reduction of the yield stress by about $10 \%$ when the short-distance interactions are omitted. This decrease is interpreted as being due to the loss of a small fraction of the forest hardening, specifically from contact reactions which do not form junctions, like the intersections of repulsive and weakly attractive dislocations. Further, a significant difference in strain hardening behavior can be observed between the two curves. When short-distance interactions are not accounted for, no strain hardening is recorded. The reason is that the dislocation density saturates shortly after yielding. In contrast, in the simulation performed without long-distance interactions, the flow stress continuously increases with plastic strain and so does, in parallel, the dislocation density. A detailed analysis of these two simulations shows that the observed differences are due to the inhibition of cross-slip activity in the absence of the short-distance interactions. Indeed, as cross-slip is a stress-assisted phenomenon, its occurrence requires the existence of high local peaks of internal stress. The latter are necessarily associated with short-distance interactions between the dislocations. Finally, and in agreement with previous simulations, it is found that dislocation patterning cannot be reproduced in conditions where the cross-slip mechanism is not sufficiently active. This can be checked by comparing the configurations of Fig. 5a and $\mathrm{b}$.

These two numerical tests have two important consequences. First, from a theoretical viewpoint, they show that in contrast with a very common belief, the onset of dislocation patterning does not seem to be related to long-distance elastic interactions. Rather, patterning is found to be governed by short-distance relaxation processes, 


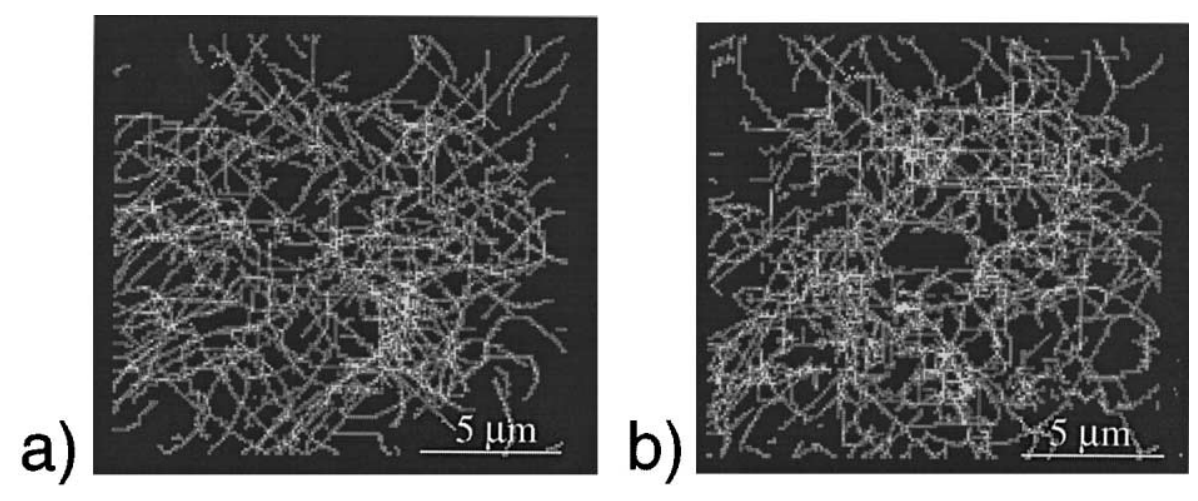

Fig. 5. Thin foils of thickness $3 \mu \mathrm{m}$ extracted from the simulations of Fig. 4 at the end of the stress-strain curve. (a) The short-distance interactions are neglected and the dislocation density is uniformly distributed in space. (b) The long-distance interactions are neglected. The dislocation distribution is no longer a random one. Notice that the diameter of the central dislocation cell is larger than the imposed cut-off value.

i.e. the cross-slip and junction formation. Secondly, from a technical point of view, one may notice that neglecting the long-distance interactions only weakly affects the mechanical response in multislip conditions. This result may significantly influence the future development of DD simulations.

\section{Approximate and exact boundary conditions}

Beyond the question of numerical efficiency, the future development of the DD simulations and their application to practical problems is conditioned by the resolution of a few theoretical problems. Among these, the most important one is without any doubt that of implementing proper boundary conditions. Numerically efficient formulations for the stress fields of straight dislocation segments exist only for the case of infinite and isotropic media. As a consequence, the boundary conditions used in most published works are not rigorous.

One can make a distinction between different levels of approximation. It is generally assumed that the loading conditions induce uniform applied stresses inside the volume of a single crystal. Then, strong approximations have to be made to account for the conditions of mechanical equilibrium at the boundaries, both in the case of small single crystals and periodic boundary conditions. The simplest possible approximation consists of considering a contact "image force" approximation, based on a modification of the dislocation line energy, when a dislocation line intersects a boundary [19]. A second level of approximation consists of solving the boundary value problem in a purely elastic manner and further, of running the DD simulation within the assumption that the contribution of the boundaries to the internal state of stress remains constant [32]. Although such an approach may look a bit oversimplified, it may apply reasonably well in one particular situation, when the plastic strain is a small fraction of the total strain. Finally, two methods have been proposed to compute exact solutions for the boundary value problem in DD simulations.
The first method makes use of the superposition principle $[33,34]$. The stress field solution of the boundary value problem is obtained as the sum of two contributions. The first one represents the solution for dislocations in an unbounded continuum and the other one is the complementary elastic solution needed to satisfy equilibrium at the external and internal boundaries. This last contribution is computed numerically by a finite element (FE) method. Such a numerical solution may seem rather simple to implement, but attention must be paid to the fact that it requires a refined measurement of the displacements at the boundaries during deformation.

The second method, which is the only one to be presently implemented in three dimensions, is based on a purely numerical technique supported by the theory of eigenstrains [35]. It does not make use of any analytical expression for the elastic field of the dislocation segments. This discrete continuous model (DCM) is based on a coupling between a DD code and a FE code, each of them functioning with its own time step (typically of $10^{-9} \mathrm{~s}$ for the DD code and $10^{-8} \mathrm{~s}$ for the FE code). On the one hand, the DD code solves the dynamics and the local reactions of discrete dislocation lines for a given state of stress in the simulated volume and yields the resulting plastic strain increment. On the other hand, the FE code computes the displacement field solution of the mechanical problem, making use of the plastic strain field yielded by the DD simulation. Thus, the DD code serves as a substitute for the constitutive form used in the usual FE framework. More precisely, the coupling procedure involves two important steps. First, the stresses defined at the nodes of the FE mesh are estimated at the positions on the dislocation lines where the dynamics is computed. Next, the discrete plastic shears produced by the motion of the dislocation segments are returned to the FE mesh. The first operation is an interpolation procedure, whereas the second one is a homogenization procedure.

The hybrid code is briefly discussed in [36-38] and its principle of operation is schematically described in Fig. 6. A FE code is used to mesh the simulated volume and 


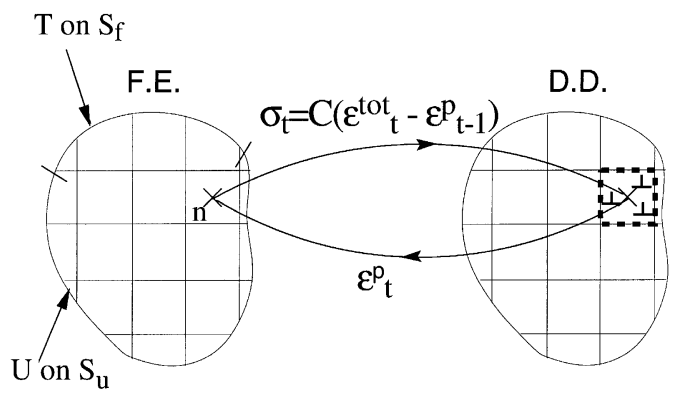

Fig. 6. Schematic drawing representing the discrete continuous model and the coupling between its FE and DD components. The mechanical problem of a dislocated body is decomposed in two parts. Left: the boundary value problem, with tensions $(\mathrm{T})$ or displacements $(\mathrm{U})$ applied to the boundaries is solved within a conventional finite element framework. Right: the constitutive law is replaced by a dislocation dynamics calculation which yields plastic strain increments, in the vicinity of each Gauss point $(n)$ of the FE mesh. The arrow from left to right represents the procedure by which the stress is interpolated at the dislocation positions. The arrow from right to left represents the homogenization of the dislocation shears at the Gauss points of the FE mesh.

periodically test the conditions of mechanical equilibrium. This FE part of the DCM, functions in a conventional manner except that, at the simulation step $t$, all the increments of total deformation are simultaneously computed at the Gauss points of the mesh elements. From the plastic strains obtained at the previous DD step, $\varepsilon_{t-1}^{\mathrm{p}}$, the stresses at step $\mathrm{t}$ are defined at the Gauss points using an explicit scheme:

$\sigma_{t}=C\left(\varepsilon_{t}^{\mathrm{tot}}-\varepsilon_{t-1}^{\mathrm{p}}\right)$

where $C$ is the tensor of elastic moduli. Notice that, in contrast with current FE formulations, the stress state has to be defined everywhere in the FE mesh at the beginning of each time step, because it serves as input for solving the dynamics in the DD part of the model. The stresses at the Gauss points of the FE mesh are interpolated to the midpoint of the dislocation segments, where the Peach-Koehler force is computed and the dislocation dynamics is solved. Once this force is estimated on all the dislocation segments, one plastic step $\Delta \varepsilon_{t}^{\mathrm{p}}$ can be performed by the DD code.

This interpolation procedure is split into two sub-steps. First the stresses defined at the Gauss points are transformed into nodal stresses by making use of the "pseudo-inverse" functions associated with the mesh elements. Such nodal stress values are common to several neighboring elements. Then, to avoid stress jumps when a dislocation segment crosses the boundary of a mesh element, an average stress value is defined at each node. The second step consists of interpolating these stresses on the dislocation segments, making use of the set of polynomial forms associated with the type of mesh element used. The quality of this interpolation is directly related to the flexibility offered by this set in terms of the number and order of the polynomials. For this reason, it is preferable to make use of meshes containing a large number of node elements.
Once the stress field interpolation has been performed, a DD step can proceed. During this step, the motion of the dislocation segments produces amounts of shear in a finite number of glide planes. These discrete plastic shears have to be transferred to the Gauss points in order to define a homogeneous plastic strain increment, $\Delta \varepsilon_{t}^{\mathrm{p}}$ that will serve as an input for the next FE step. This homogenization procedure, which is an essential part of the coupling between the two codes, will not be discussed here by lack of space. The basic problem consists of finding an adequate volume within which the individual areas swept by the dislocation lines can be transformed into local average shears (cf. [38] for more details). When the plastic strain increment is known, the conditions of mechanical equilibrium are tested by the FE code and, if necessary, the whole procedure described above is iterated until a prescribed level of convergence is met. The number of iterations needed at each step of DCM depends, of course, on the complexity of the investigated configuration, on the amplitude of the time or deformation increment tested and the type of FE mesh element used. As an example, the number of iterations performed in the example given below is always smaller than five.

Although the DCM procedure is complex in technical terms, it is physically transparent. In contrast with the traditional constitutive formulations, the DCM makes use of a restricted number of state variables, principally the stress tensor and the elastic and plastic strain tensors. The latter incorporates all the physical properties of the defects that are included into the DD code, in particular it includes microstructural length scales. The stress fields of the dislocation segments is no longer an input property of the DD code, it is naturally obtained as part of the global solution of the mechanical problem. Thus, the computation of the stress on dislocation segments being interpolated from a constant number of nodes in the FE mesh, the calculation of the dislocation-dislocation elastic interactions is no longer an $\mathrm{O}\left(\mathrm{N}^{2}\right)$ problem. Finally, one may notice that taking into account the long range character of the dislocation fields is strictly equivalent to making use of a non-local constitutive form.

To conclude this section, it must be emphasized that the above numerical method, or its equivalents making use of the superposition principle, provide an exact mechanical solution for crystals of finite dimensions at the boundaries of which dislocations are blocked (interfaces, grain boundaries) or eliminated (free surfaces). However, they only offer a partial solution to the case of infinite crystals computed with the help of periodic boundary conditions. Indeed, if equilibrium conditions can be computed without difficulty by FE methods, the complete solution also requires the balance between the dislocation fluxes leaving and entering the surfaces of the elementary crystal to be duplicated by the boundary conditions. This last condition is quite difficult to implement in $3 \mathrm{D}[39,40]$ and work on it is still in progress. 


\section{Dislocation motion in the channels of superalloys}

The DCM presented in the previous section involves intensive numerical calculations, as a large number of 3D FE meshes is required for a precise interpolation of the dislocation self-stress fields. For this reason, problems of confined plasticity that cannot be solved by classical DD or FE simulations alone have been examined first. There are, in practice, many situations where dislocation motion is confined to a small volume bounded by interfaces. In such cases, non-unifom internal stress fields are involved and size effects manifest themselves, due to either line tension or pile-up effects. The DCM has been applied to two typical examples, the plastic relaxation of internal stresses in thin epitaxial layers and in channels bounded by two interfaces. The case of thin films is discussed in [36]. In what follows, we consider the calculation of the critical stress needed to propagate a single dislocation in a $\gamma$ channel of a $\gamma / \gamma^{\prime}$ superalloy.

Nickel-base superalloys have a biphased structure composed of cuboidal particles of a $\mathrm{L}_{2}$ ordered phase based on $\mathrm{Ni}_{3} \mathrm{Al}$, the $\gamma^{\prime}$-phase, embedded into a disordered matrix of similar composition, the $\gamma$-phase (cf. Fig. 7). The lattice parameters and thermal expansion coefficients of the two phases differ, leading to misfit stresses at the coherent interfaces between the two phases. It is usually assumed that plasticity mainly occurs in the disordered phase at high temperatures. The elastic constants slightly differ in the two phases, leading to weak incompatibility stresses in the elastic regime but strong incompatibility stresses as soon as the $\gamma$-phase becomes plastic. The interplay between these complex internal stresses, the applied stress and dislocation behavior has not yet been completely cleared up, as it necessitates a combination of discrete and continuum modeling. The present state of understanding of the plasticity of these materials has been summed up recently by Nabarro and de Villiers [41].

When a tensile stress is applied along the [0 001$]$ direction, all the $\left\{\begin{array}{lll}1 & 0 & 0\end{array}\right\}$ interfaces are not equivalent (Fig. 7). The

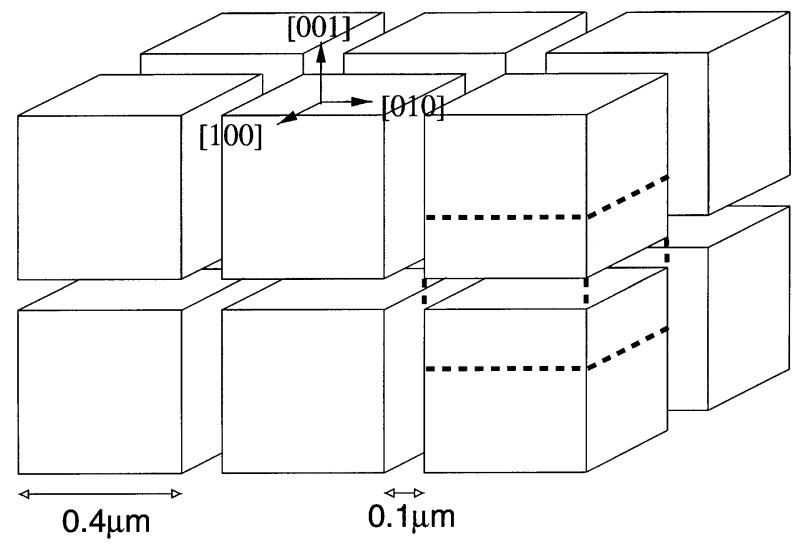

Fig. 7. Schematic view of the microstructure of a $\gamma / \gamma^{\prime}$ nickel-based superalloy with high volume fraction of cuboidal $\gamma^{\prime}$ precipitates. The dotted lines represent the volume simulated by the DCM.
Table 1

Elastic constants and lattice parameter of the MC2 superalloy, as used in the DCM calculations

\begin{tabular}{lcc}
\hline Constants at $1050^{\circ} \mathrm{C}$ & $\gamma$-Phase & $\gamma^{\prime}$-Phase \\
\hline$C_{11}(\mathrm{GPa})$ & 129 & 162 \\
$C_{12}(\mathrm{GPa})$ & 65 & 78 \\
$C_{44}(\mathrm{GPa})$ & 182 & 222 \\
$a(\mathrm{~nm})$ & 0.3578 & \\
\hline
\end{tabular}

horizontal $(001)$ interface is normal to the applied stress and the interfaces of the corresponding channels are loaded in tension ( $\mathrm{T}$ type). The (100) and (0 100$)$ interfaces are parallel to the tensile axis and the corresponding interfaces are in tension (C type). Accordingly, and in agreement with experimental measurements, the nature of the active slip systems and the local plastic strains largely differ in the two types of channels. They further depend upon the mode of loading, tension or compression.

The objective of the calculations reported below is to estimate the maximum difference in yield stresses between channels whose interfaces are loaded in tension or in compression. For this purpose, the DCM simulation was used to compute the critical stress needed to move a single dislocation on a given slip system, in a channel loaded either in tension or in compression. This simulation was performed in a channel free of misfitting dislocations, as is usually the case at the beginning of plastic flow. The geometry of the DCM calculations is depicted in Fig. 8 and the dimensions of the simulated volume are given in Fig. 7. The elastic constants used for the computations and the lattice parameter of the $\gamma$-phase are given in Table 1 . They correspond to the MC2 superalloy which has been developed at ONERA. The FE mesh is made up of 1536 quadratic elements, 20 nodes and 8 Gauss points (1024 elements in the $\gamma$-phase and 512 in $\gamma^{\prime}$-phase). Before any applied stress is imposed, the FE mesh is loaded by imposing different thermal expansions to the two phases. This preliminary loading is fitted to reproduce a misfit of $-0.3 \%$ at the interfaces, i.e. the value experimentally measured at $1050^{\circ} \mathrm{C}$ [42]. A half-dislocation loop of Burgers vector, $b=a / 2\left[\begin{array}{lll}1 & 0 & 1\end{array}\right]$ gliding in a (1 111$)$ plane, is then introduced through one side of the simulated volume (cf. Fig. 8a) thus, setting the initial conditions for the DCM simulations.To determine the critical stress for dislocation motion in the T and C channels, the $\gamma^{\prime} / \gamma / \gamma^{\prime}$ composite is loaded with the following displacement rates (cf. Fig. 8): $(0,0,-\dot{u})$ on the lower face and $(0,0, \dot{u})$ on the upper face for the channels of T type, $(0,0, \dot{u})$ on the lower face and $(0,0,-\dot{u})$ on the upper face for the channels of $\mathrm{C}$ type, with $\dot{u}=1.8 \times 10^{-3} \mu \mathrm{m} / \mathrm{s}$. The computations were stopped when a critical stress was reached such that the initial dislocation segment started to move into the channel, depositing segments of mixed character at the interfaces.

The difference in yield stress recorded between tension and compression is amazingly large and clearly illustrates the importance of a correct description of the internal stress 

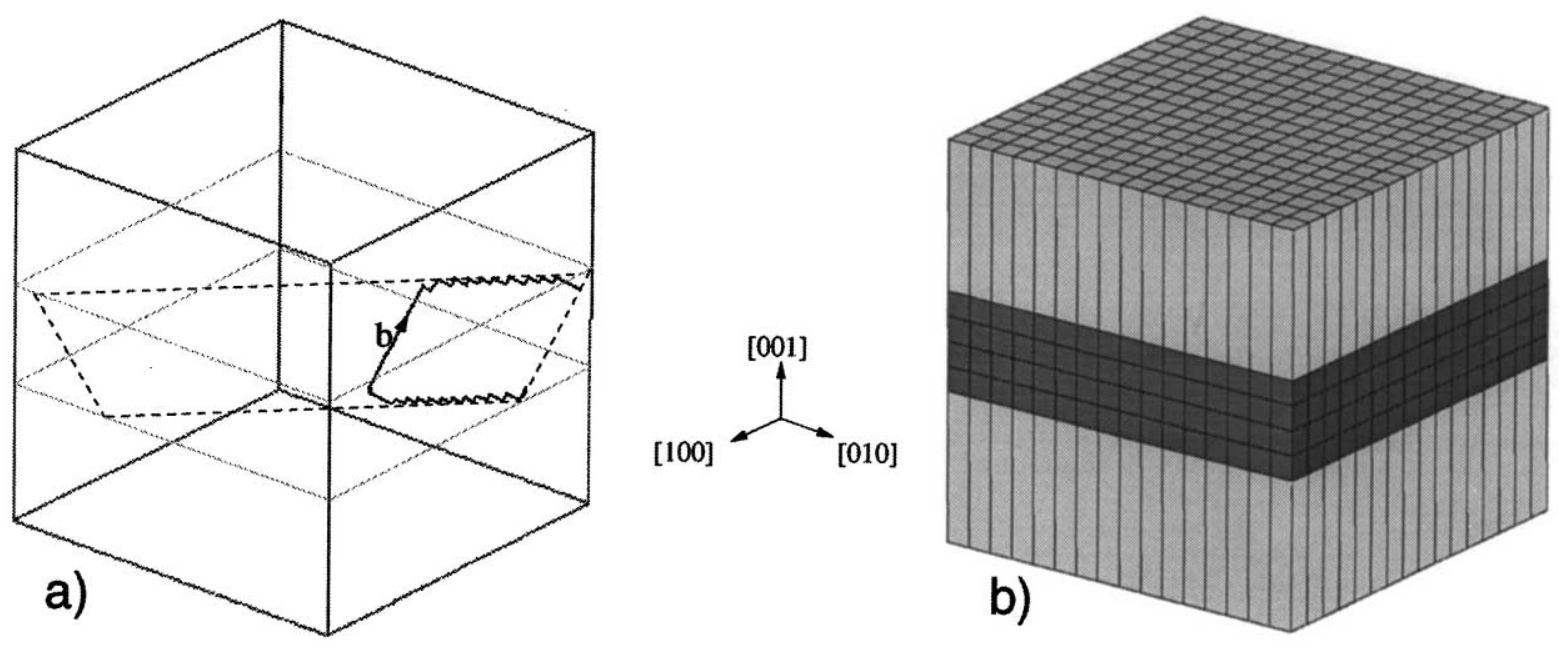

Fig. 8. The geometry of the DCM calculation. (a) The simulation box with a single dislocation being introduced from left to right into a (111) slip plane bounded by the two $\gamma / \gamma^{\prime}$-interfaces. (b) The corresponding FE mesh, showing the different types of meshing used for the elastic $\gamma^{\prime}$-phase and the plastic $\gamma$-phase.

fields during dislocation dynamics calculations. Indeed, for the considered Burgers vector and slip system, a critical resolved stress of $185 \mathrm{MPa}$ is found in compression, whereas only $105 \mathrm{MPa}$ are needed in tension. The analysis of the stress mappings in the dislocation glide plane straightforwardly explains the observed effect. In the channel loaded in tension, the stress field of the dislocation line deposited at the $\gamma / \gamma^{\prime}$-interfaces relaxes the misfit stress. In the channel loaded in tension, it enhances the misfit stress. Equivalently, one can say that in the channels of type $\mathrm{T}$, the effect of the



Fig. 9. Mapping of the resolved stresses in the dislocation glide plane. When the channels are dislocation-free, the misfit at the $\gamma / \gamma^{\prime}$-interfaces induces the same internal stress of about $-150 \mathrm{MPa}$ in the channels loaded in tension ( $\mathrm{T}$ ) or compression (C). In the area sheared by the dislocation, this internal stress is increased in the $\mathrm{C}$ channel and decreased in the $\mathrm{T}$ channel. These internal stress mappings are drawn under practically no applied stress, at the very beginning of the loading sequence. internal stress on the moving dislocations adds up to that of the applied stress, while it is resistive in the channels of type $\mathrm{C}$. These results can be extended to predict the active slip planes in the two types of channels, in tension and in compression, and the resulting asymmetries (Fig. 9).

\section{Concluding remarks}

As shown in this short review, the methods for simulating dislocation dynamics at the mesoscale are the object of continuous improvements. Whereas they are all based on the same elastic framework, different solutions have been proposed in order to discretize the dislocation lines, which have their own strengths and weaknesses. In this respect, the pure-mixed model presented here offers an excellent compromise between computing efficiency and numerical accuracy.

A careful treatment of dislocation core and contact properties or reactions, viz. dislocation mobility, cross-slip and the formation and destruction of junctions, is a prerequisite for any consistent study on dislocation-based plasticity. This point is illustrated by the simulations of dislocation cell formation in fec crystals strained in multiple slip conditions. The conclusion drawn from this study is that current modeling overestimates the role of long range stresses in dislocation pattern formation and underestimates the role of short range interactions and local mechanisms.

Finally, the most important challenge for the future of mesoscopic simulation consists of implementing a rigorous treatment of the boundary conditions in order to go beyond the now traditional studies on single crystals. This necessarily involves a coupling with a continuous framework, which is now under way, and will lead to two types of developments. The new hybrid simulations will allow 
treating classical problems in mechanical engineering and, above all, they may be useful to reconcile the continuum and discrete approaches of plasticity.

\section{Acknowledgements}

The authors are grateful to Jean-Louis Chaboche (ONERA-DMSE) for helpful discussions on the discretecontinuum connection.

\section{References}

[1] A.J.E. Foreman, M.J. Makin, Phil. Mag. 14 (1966) 911.

[2] D. Bacon, Phys. Stat. Sol. 23 (1967) 527.

[3] D. Bacon, U.F. Kocks, R.O. Scattergood, Phil. Mag. 28 (1973) 1241.

[4] L.P. Kubin, G.R. Canova, The formation of ordered dislocation microstructures, in: U. Messerschmidt, et al. (Eds.), Electron Microscopy in Plasticity and Fracture Research of Materials, Akademie Verlag, Berlin, 1990, pp. 23-32.

[5] L.P. Kubin, G.R. Canova, Scripta Metall. Mater. 27 (1992) 957.

[6] L.P. Kubin, G.R. Canova, M. Condat, B. Devincre, V. Pontikis, Y. Bréchet, Solid State Phenom. 23/24 (1992) 455.

[7] B. Devincre, L.P. Kubin, Mater. Sci. Eng. 2 (1994) 559.

[8] L.P. Kubin, B. Devincre, M. Tang, J. Comput. Aided Mat. Design 5 (1998) 31.

[9] M. Tang, B. Devincre, L.P. Kubin, Mat. Sci. Eng. 7 (1999) 893.

[10] B. Devincre, S. Roberts, Acta Metall. 74 (1996) 2891.

[11] M. Fivel, C.F. Robertson, G.R. Canova, L. Boulanger, Acta Mater. 46 (1998) 6183.

[12] A. Moulin, M. Condat, L.P. Kubin, Acta Mater. 47 (1999) 2870.

[13] B. Devincre, P. Veyssière, L.P. Kubin, G. Saada, Phil. Mag. A 75 (1997) 1263.

[14] K.W. Schwarz, J. Appl. Phys. 85 (1999) 120.

[15] L.K. Wickham, K.W. Schwarz, J.S. Stölken, Phys. Rev. Lett. 83 (1999) 4574.

[16] V.B. Shenoy, R.V. Kukta, R. Phillips, Phys. Rev. Lett. 84 (2000) 1491.

[17] N.M. Ghoniem, B.N. Singh, L.Z. Sun, T. Diaz de la Rubia, J. Nuclear Mater. 276 (2000) 166.
[18] H.M. Zbib, T. Diaz de la Rubia, M. Rhee, J.P. Hirth, J. Nuclear Mater. 276 (2000) 154.

[19] B. Devincre. Mesoscale simulation of the dislocation dynamics, in: H.O. Kirchner, V. Pontikis, L.P. Kubin (Eds.), Computer Simulation in Materials Science, North-Holland, Amsterdam, 1996, p. 309.

[20] M. Verdier, M. Fivel, I. Groma, Modelling Simul. Mater. Sci. Eng. 6 (1998) 755.

[21] H.M. Zbib, M. Rhee, J.P. Hirth, Int. J. Mech. Sci. 40 (1998) 113.

[22] K.W. Schwarz, I. Methods and example, J. Appl. Phys. 85 (1999) 108.

[23] N.M. Ghoniem, S.H. Tong, L.Z. Sun, Phys. Rev. B 139 (2000) 913.

[24] A. Moulin, M. Condat, L.P. Kubin, Acta Mater. 45 (1997) 2339.

[25] M. Tang, L.P. Kubin, G.R. Canova, Acta Mater. 46 (1998) 3221.

[26] B. Devincre, M. Condat, Acta Metall. Mater. 40 (1992) 2629.

[27] D. Rodney, R. Phillips, Phys. Rev. Lett. 82 (1999) 1704.

[28] M. Rhee, H.M. Zbib, J.P. Hirth, K. Huang, T. Diaz de la Rubia, Modelling Simul. Mater. Sci. Eng. 6 (1998) 467.

[29] B. Devincre, L.P. Kubin, Mat. Sci. Eng. A 234-s236 (1997) 8.

[30] L.P. Kubin, B. Devincre, From dislocation mechanisms to dislocation microstructures and strain hardening, in: J.B. Bilde-Sorensen, et al. (Eds.), Deformation-Induced Microstructures: Analysis and Relation to Properties, Risoe National Laboratory, Roskilde, Denmark, 1999, pp. 61-83.

[31] P.J. Jackson, Prog. Mater. Sci. 29 (1985) 139.

[32] X.H. Liu, F.M. Ross, K.W. Schwarz, Phys. Rev. Lett., 2000, submitted for publication.

[33] H.H.M. Cleveringa, E. van der Giessen, A. Needleman, Acta Mater. 45 (1997) 3163.

[34] M. Fivel, A. EI-Azab, J. de Phys. IV 9 (1999) 278.

[35] T. Mura, Micromechanics of defects in solids, Kluwer Academic Publishers, Dordrecht, 1993.

[36] C. Lemarchand, J.L. Chaboche, B. Devincre, L.P. Kubin, J. de Phys. IV 9 (1999) 271.

[37] C. Lemarchand, B. Devincre, L.P. Kubin, J.L. Chaboche, MRS Symp. Proc. 538 (1999) 63.

[38] C. Lemarchand, B. Devincre, L.P. Kubin, J. Mech. Phys. Solids, submitted for publication.

[39] A. El-Azab, Modelling Simul. Mater. Sci. Eng., 2000, in press.

[40] M. Fivel, G.R. Canova, Modelling Simul. Mater. Sci. Eng. 7 (1999) 753.

[41] F.R.N. Nabarro, H.L. de Villiers, The physics of creep, Taylor \& Francis, London, 1995.

[42] D. Nouailhas, G. Cailletaud, Scripta Mater. 34 (1995) 565. 